\title{
RAMAN SCATTERING BY PLASMONS IN FIBONACCI SUPERLATTICES
}

\author{
R. Merlin \\ The Harrison M. Randall Laboratory of Physics, The University of Michigan, \\ Ann Arbor, MI 48109-1120, USA
}

J. P. Valladares, A. Pinczuk, A. C. Gossard ${ }^{*}$ and J. H. English* AT\&T Bell Laboratories, Murray Hill, NJ 07974-0636, USA

(Received 28 May 1992 by M. Cardona)

\begin{abstract}
We report on resonant Raman scattering by plasmons in a modulation-doped Fibonacci superlattice consisting of $270-A$-thick $\mathrm{GaAs}$ wells separated by $\mathrm{Al}_{0.3} \mathrm{Ga}_{0.7} \mathrm{As}$ barriers whose thickness is either $800 \mathrm{~A}$ or $430 \mathrm{~A}$. The ordering of the barriers is determined by the Fibonacci sequence. In the region below $\sim 10 \mathrm{meV}$, the spectra exhibit relatively broad features (full width $\approx 0.5 \mathrm{meV}$ ) resulting from a large number of unresolved plasmon contributions. The dependence of the peak positions on the in-plane wavevector is in good agreement with theoretical predictions.
\end{abstract}

ELEMENTARY EXCITATIONS in quasiperiodic systems and, in particular, Fibonacci superlattices, have been the object of intensive theoretical study in recent years. ${ }^{1}$ The generic Fibonacci spectrum, characterized by critical (power-law decaying) eigenstates and a fractallike structure of gaps, ${ }^{1}$ distinguishes itself dramatically from the Bloch's behavior of periodic solids. Experimentally, most of the work on excitations has concentrated on acoustic phonons in $\mathrm{Al}_{\mathrm{x}} \mathrm{Ga}_{1-\mathrm{x}} \mathrm{As}$ and $\mathrm{Ge}_{\mathrm{x}} \mathrm{Si}_{1-\mathrm{x}}$ superlattices. ${ }^{1} \mathrm{Be}-$ cause acoustic modes in these systems are hardly perturbed by the layering, however, the measurements (especially those of Raman scatte-ing) ${ }^{2}$ bear much more on quasiperiodic structural aspects than on the properties of the phonons. In this communication, we concern ourselves with plasmons in a Fibonacci layered electron system. ${ }^{3-5}$ Unlike acoustic phonons, plasmon eigenfunctions depend strongly on the parameters and the sequence defining the superlattice. ${ }^{3-5}$ Our study was partially motivated by theoretical results comparing Raman spectra of plasmons in periodic and quasiperiodic structures. ${ }^{3}$ A preliminary account of our data is given in Ref. $2 .^{6}$

The structure used in this work was a modulation-doped Fibonacci superlattice grown by

\footnotetext{
Present address: Materials Department, University of California - Santa Barbara, Santa Barbara, CA 93106, U. S. A.
}

molecular beam epitaxy on a (100) GaAs substrate. The sample is defined 1,2 by the ninth generation of the Fibonacci sequence of 270- $\AA$-thick GaAs/800- $\AA$-thick $\mathrm{Al}_{0.3} \mathrm{Ga}_{0.7} \mathrm{As}$ (building block $A$ ) and $270-\AA$-thick GaAs/430-A-thick $\mathrm{Al}_{0.3} \mathrm{Ga}_{0.7} \mathrm{As}$ (building block $B$ ). Alternatively, there is a total of 55 GaAs wells (all 270-Athick) separated by either $d_{A}=1070 \AA$ or $d_{B}=700 A$ (distances between well centers) in a pattern determined by the Fibonacci rule $S_{r}=S_{r-1} S_{r-2}\left(S_{r}\right.$ is the rth-generation sequence, with $S_{1} \equiv d_{A}$ and $\left.S_{2} \equiv d_{A} d_{B}\right)$. Transmission electron microscopy was used to determine the thicknesses of the layers. The $\mathrm{Al}_{0.3} \mathrm{Ga}_{0.7} \mathrm{As}$ barriers were doped with Si-donors leading to trapping of carriers by the GaAs wells and, thus, to the formation of a set of 55 quasi-two-dimensional electron systems. We chose a doping profile expected to give the same free electron density $(n)$ across the superlattice. From the analysis of photoluminescence data, we find the average value $n=3.8 \times 10^{11} \mathrm{~cm}^{-2}$ corresponding to two occupied subbands.

Raman measurements were performed at $\approx 4 \mathrm{~K}$ using an LD700 dye-laser pumped by a $\mathrm{Kr}$-laser. The LD700 dye operates in the range 7800-8000 $\AA$ covering the region of resonances with (quantumwell) critical points derived from the fundamental gap of GaAs. Resonant mechanisms for elec- 
tronic Raman scattering in quasi-two-dimensional systems - particularly GaAs surface layers were first considered by Burstein et al. ${ }^{7}$ Spectra with a resolution of $\approx 2 \mathrm{~cm}^{-1}$ were obtained in the standard backscattering geometry. Scattering by plasmons is allowed when the polarizations of the incident and scattered photons are parallel, irrespective of the orientation of the polarization with respect to crystal axes. 8 The magnitude of the in-plane scattering wavevector $q$ was varied by changing the angle $\theta$ between the laser beam and the normal to the layers ( $q=4 \pi \sin \theta / \lambda_{\mathrm{L}}$, where $\lambda_{\mathrm{L}}$ is the laser wavelength). 8 In our experiments, we were able to probe in-plane wavevectors in the range $0.1-$ $1.5\left(\times 10^{5}\right) \mathrm{cm}^{-1}$. The corresponding range for the component normal to the layers, $k$, is $5-7$ (x $\left.10^{5}\right) \mathrm{cm}^{-1}$.

In the calculations, the induced potential at the lth-well $\phi_{\nu}(l)$ and the frequency $\omega_{\nu}$ of the plasmons were obtained by numerically solving the tight-binding equation $3-5,9$

$$
\left(\omega_{v}^{2} / \Omega_{\mathrm{p}}^{2}-1\right) \phi_{v^{\prime}}(l)=\sum_{l \neq l^{\prime}} \phi_{v^{\prime}}\left(l^{\prime}\right) v\left(l, l^{\prime}\right),
$$

where $v$ labels the eigenstates. Here, $\Omega_{p}(q)=$ $\left(2 \pi e^{2} n q / \varepsilon m_{0}\right)^{1 / 2}$ is the two-dimensional plasma frequency and $V\left(l, l^{\prime}\right)=\exp \left(-q\left|z_{l}-z_{l},\right|\right) ; z_{l}$ is the position of the center of the lth-well, $m_{0}$ is the electron effective mass and $\varepsilon$ is the background dielectric constant. Eq. (1) describes plasmons propagating parallel to the layers with a wavevector of magnitude $q$. The scattering intensity $I$ was calculated using the expression which relates it to the dynamical structure factor of the system?

$$
I(\omega, q, k) \propto \sum_{v}\left|\phi_{v}(k)\right|^{2} \delta\left(\omega-\omega_{\nu}\right)
$$

with $\phi_{\nu}(k)=\sum \exp \left(i k z_{l}\right) \phi_{\nu}(l)$ (as before, $k$ is the normal component of the scattering wavevector). Broadening was accounted for by introducing a phenomenological damping parameter $\gamma$ so that the $\delta$-function is replaced by the expression $1 /\left[\left(\omega-\omega_{v}\right)^{2}+\gamma^{2}\right]$.

Figure 1(a) shows low-energy Raman spectra for various values of the in-plane scattering wavevector $q$. The calculated dispersion of plas-
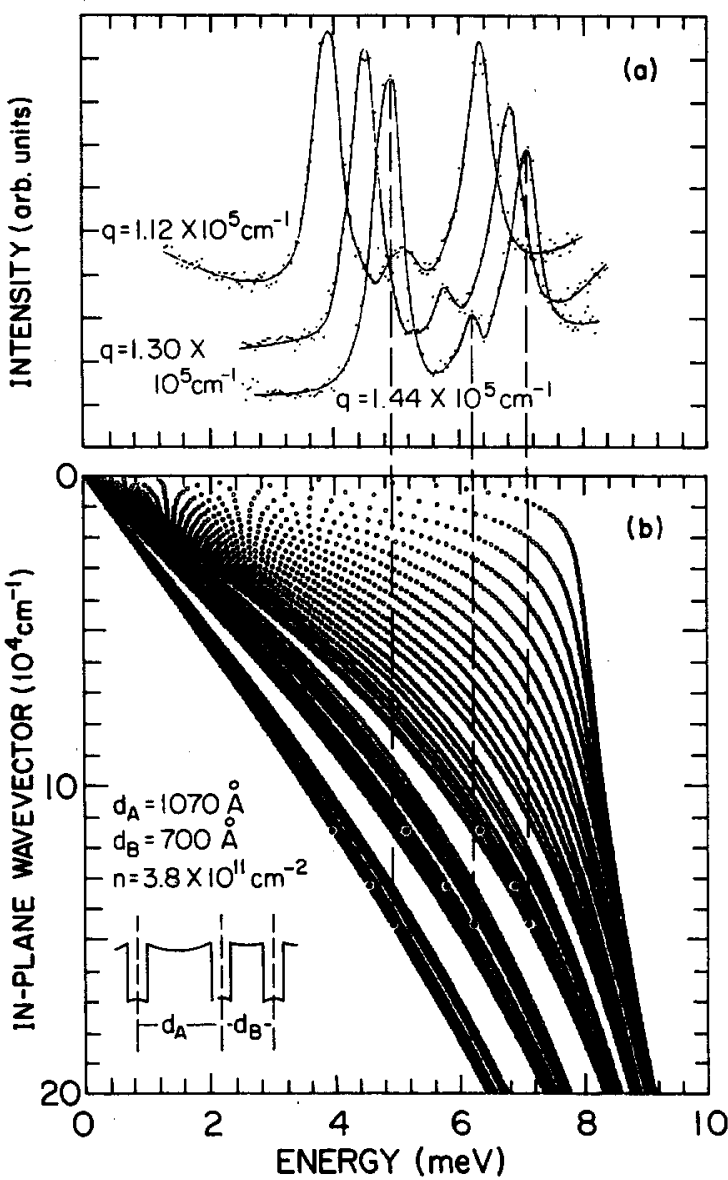

Figure 1. (a) Raman spectra showing scattering by plasmons, $q$ is the in-plane component of the scattering wavevector. The laser energy is $1.569 \mathrm{eV}$. (b) Calculated dispersion of plasma modes. Dots represent the measured maxima of spectral features. Inset: Sample parameters.

ma modes is reproduced in Fig. 1(b); notice that the number of solutions to Eq. (1) at a given $q$ is equal to the number of GaAs wells. As shown by the dashed lines connecting Figs. $l(a)$ and $1(b)$, the positions of the Raman peaks - dots in Fig. 1(b) - correlate with calculated plasmon eigenenergies. Results of numerical simulations using Eq. (2) are shown in Fig. 2(a). The comparison with the experimental data strongly support our identification of the scattering in Fig. 1(a) as due to plasmons. Furthermore, the observed $\gamma$-behavior of the theoretical spectra indicates that the three main lines consist of much narrower features separated by $\sim 0.1-0.3$ meV. In Fig. 2(b), we compare the calculated and measured positions of these features. The 

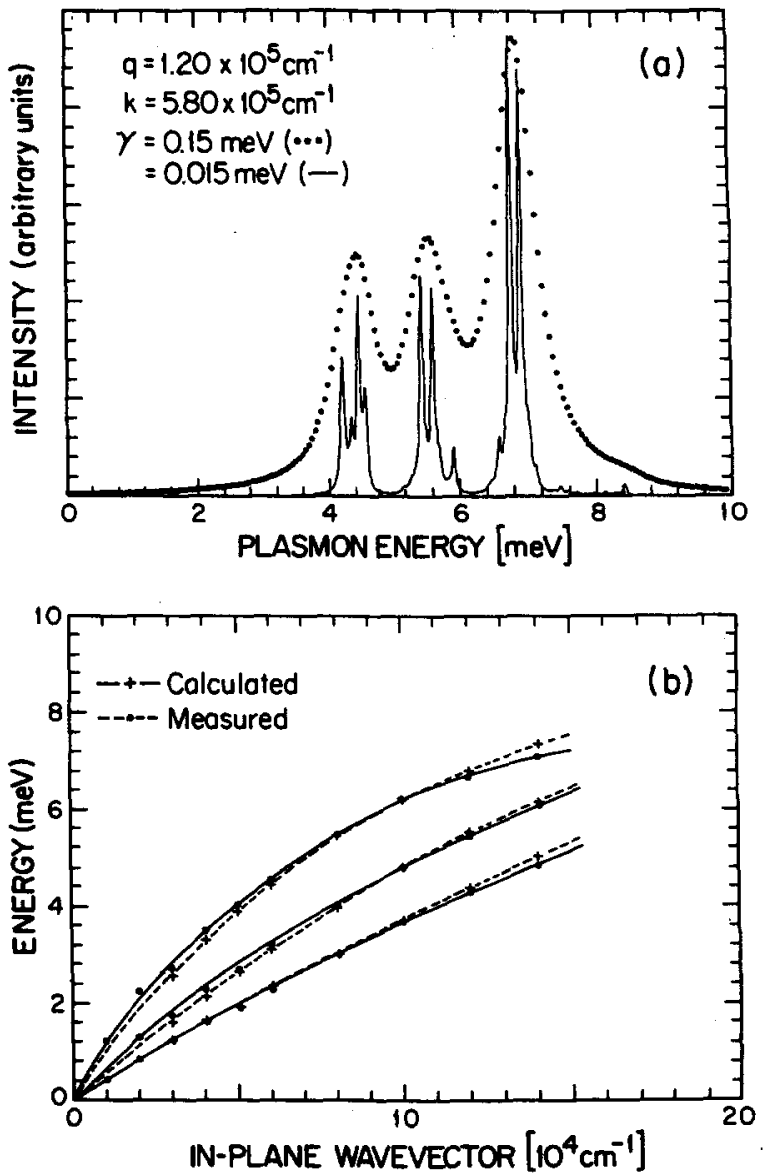

Figure 2. (a) Calculated Raman intensity at two different linewidths. (b) Comparison between calculated and measured dispersions. Dashed and full curves are guides for the eye.

calculations agree reasonably well with the measured $q$-dependence of the Raman shifts, but do not account for the relative intensities of the major peaks. It is not clear whether this problem reflects difficulties with Eq. (2) or an experimental artifact due to departures from norninal values of the sample parameters.

In recognition of his fundamental work in the field of Raman scattering and his profound influence on many generations of students (and students of students), it is a pleasure to dedicate this paper to Professor Elias Burstein on the occasion of his 75th birthday.

Acknowledgments - We are very grateful to Don Werder for expert TEM characterization of the sample. This work was supported in part by the U. S. Army Research office under contract No. DAAL03-92-G-0233.

\section{REFERENCES}

1. For reviews, see: M. Kohmoto, Int. J. Mod. Phys. B 1, 31 (1987); R. Merlin, IEEE J. Quantum Electron. 24, 1791 (1988); A. H. MacDonald, in Interfaces, Quantum Wells and Superlattices, ed. by C. R. Leavens and R. Taylor (Plenum Press, New York, 1988), p. 347.

2. For a review, see: $R$. Merlin, in Light Scattering in Solids $V$, ed. by M. Cardona and G. Güntherodt, Topics Appl. Phys. 66 (Springer, Berlin, 1989), p. 214.

3. S. Das Sarma, A. Kobayashi and R. E. Prange, Phys. Rev. B 34, 5309 (1986).

4. P. Hawrylak and J. J. Quinn, Phys. Rev. Lett. 57, 380 (1986).

5. D. Huang, J. Peng and S. Zhou, Phys. Rev. B 40, 7754 (1989).

6. The component of the wavevector appearing in Fig. 12 and Eq. (20) of Ref. 2 (p. 229) should be the parallel $(q)$ instead of the perpendicular $(k)$ one.

7. E. Burstein, A: Pinczuk and S. Buchner, in Physics of Semiconductors 1978 , ed. by B. H. L. Wilson, Conference Series 43 (The Institute of Physics, Bristol, 1979), p. 1231.

8. A. Pinczuk and G. Abstreiter, in Light Scattering in Solids $V$, ed. by $M$. Cardona and G. Güntherodt, Topics Appl. Phys. 66 (Springer, Berlin, 1989), p. 153.

9. J. K. Jain and P. B. Allen, Phys. Rev. B 32, 997 (1985). 